Orijinal Araștırma

\title{
Sikatrisyel alopeside dermoskopik bulgular
}

\section{Dermoscopic findings in cicatricial alopecia}

\section{Seher Arı, Gonca Gökdemir*}

Gebze Fatih Devlet Hastanesi, Dermatoloji Kliniği, Kocaeli, Türkiye

*Liv Hospital, Dermatoloji Kliniği, İstanbul, Türkiye

\section{Özet}

Amaç: Dermoskopi pigmente deri lezyonlarının tanısında önemli bir araçtır. Son yıllarda bu yöntem saç ve saçı deri hastalıklarının tanı ve takibinde de kullanılmaktadır. Çalışmanın amacı klinik ve histopatolojik olarak sikatrisyel alopesisi olan bir grup hastada dermoskopik bulguları değerlendirmekti.

Gereç ve Yöntem: Klinik ve histolojik bulgulara dayanarak sikatrisyel alopesi tanııı konulan 29 hasta dermoskopik özellikler açısından değerlendirildi.

Bulgular: Çalışmaya katılan folikülitis dekalvanslı 8, Brocq'un psödopeladı olan 7, liken planopilarisli 6, diskoid lupus eritematozuslu 2 , dissekan selüliti olan 1 ve sekonder skatrisyel alopesili 5 hastada dermoskopik özellikler incelendi. Çıplak gözle muayenede görülen özellikler dermoskopi ile daha detaylı değerlendirildi. Sikatrisyel alopesili hastaların hepsinde foliküler açıklık kaybı vardı. Perifoliküler skuam, dallanan kırmızı çizgiler, bal peteği pigment paterni, beyaz benekler ve tufted folikülit diğer sık görülen bulgulardı.

Sonuç: Sikatrisyel alopesinin klinik değerlendirilmesinde dermoskopinin kullanımı, basit klinik inspeksiyonun ötesinde tanısal yararlıık sağlar, fakat bu yöntemin daha iyi anlaşıması için histopatolojik korelasyonun da yapııldı̆ı geniş serili çalışmalara ihtiyaç vardır. (Türkderm 2013; 47: 223-6)

Anahtar Kelimeler: Sikatrisyel, alopesi, dermoskopi

\section{Summary}

Background and Design: Dermoscopy is an important tool in the diagnosis of pigmented skin lesions. Newly, this method has also been used in the diagnosis and follow-up of hair and scalp disorders. The objective of this study was to investigate dermoscopic findings in a sample of patients diagnosed with cicatricial alopecia based on clinical and histopathological findings.

Materials and Methods: Twenty-nine patients with cicatricial alopecia diagnosed according to the clinical and histological findings were examined by dermoscopy.

Results: Dermoscopic features were evaluated in 8 patients with folliculitis decalvans, - 7 with pseudopelade of Brocq, - 6 with lichen planopilaris, - 2 with discoid lupus erythematosus, - 1 with dissecting cellulitis, and in 5 patients with secondary cicatricial alopecia. The structures previously examined with naked eye were evaluated by dermoscopy in more detail. Loss of follicular orifices was seen in all patients with cicatricial alopecia. Perifollicular scaling, arborizing red lines, honeycomb pigment pattern, white dots and tufted hairs were the other most obvious findings.

Conclusion: The use of dermoscopy in the clinical evaluation of cicatricial alopecia improves diagnostic capability beyond simple clinical inspection. Larger studies correlating dermoscopic findings with histopathology examinations are necessary to improve our understanding of this method. (Turkderm 2013; 47: 223-6)

Key Words: Cicatricial, alopecia, dermoscopy

Yazışma Adresi/Address for Correspondence: Dr. Seher Arı, Gebze Fatih Devlet Hastanesi, Dermatoloji Kliniği, Kocaeli, Türkiye E-posta: dr_seherari@yahoo.com Geliş Tarihi/Received: 19.07.2012 Kabul Tarihi/Accepted: 24.08.2011

Türkderm-Deri Hastalıkları ve Frengi Arșivi Dergisi, Galenos Yayınevi tarafından basılmıștır. Turkderm-Archives of the Turkish Dermatology and Venerology, published by Galenos Publishing. 


\section{Giriş}

Skatrisyel alopesi kıl foliküllerinin kalıcı hasarıyla karakterize inatçı seyreden ve az görülen ayrı bir inflamatuvar hastalık grubudur. Primer skatrisyel alopesi öncelikli olarak pilosebase üniteyi hedef alan kronik inflamatuvar bir süreç olmasına rağmen sekonder tipte spesifik olarak folikülosentrik tutulum yoktur. Primer veya sekonder olsun tüm skatrisyel alopesilerde klinik olarak foliküler açıklık kaybı ve histolojik olarak kıl folikülünün fibröz doku ile yer değiştirmesiyle karakterize kalıcı hasar mevcuttur ${ }^{1-4}$. Skatrisyel alopeside klinik muayene her zaman tam bilgi vermez. Dermoskopi son yıllarda saç ve saçlı deri hastalıklarının tanı ve takibinde kullanılmaktadır5-7. Dermoskopik olarak tanımlanmış spesifik paternler saç ve saçlı deri hastalıklarının ayırıcı tanısında klinisyene yardımcı olabilir. Literatürde skatrisyel olmayan alopeside dermoskopik bulgularla ilgili çeşitli çalışmalar vardır. Skatrisyel alopeside ise olgu sunumları ve küçük serili çalışmalar bildirilmiştir.

Biz bu çalısmada, klinik ve histopatolojik olarak skatrisyel alopesisi olan bir grup hastada dermoskopik bulguları analiz etmeyi ve dermoskopinin skatrisyel alopesi tanısında yararlıı̆ını değerlendirmeyi amaçladık.

\section{Gereç ve Yöntem}

Bu çalışma Ekim 2009-Nisan 2010 tarihleri arasında dermatoloji polikliniğimizde yürütüldü. Yirmi dokuz skatrisyel alopesisi olan hasta çalışmaya alındı. Tüm hastalarda tanı anamnez, klinik görünüm ve histopatolojik değerlendirme ile konuldu. Hastaların demografik özellikleri (yaş, cinsiyet), yakınmaları, hastalık süresi ve tedavi öyküsü kayıt edildi. Saçlı derideki alopesik alanların dermoskopik değerlendirmesi Dermlite II pro dermoskop (3Gen) kullanılarak yapıldı. Dermoskopik görüntüler Dermlite Pro II'ye bağlı dijital kamera (Nikon coolpix-4500) kullanılarak fotoğraflandı. Tüm görüntüler her iki araştırmacı tarafından dermoskopik özellikler (vasküler yapılar, deri yüzeyinin görünümü ve foliküler/perifoliküler bulgular) açısından değerlendirildi.

\section{Bulgular}

Çalışmaya $15^{\prime} i$ kadın, 14 'ü erkek toplam 29 skatrisyel alopesili hasta katıldı. Hastaların yaş ortalaması 31,31 (3-57) idi. Hastalık süresi 3 ay ile 20 yıl arasında değişmekteydi. Hastaların en sık yakındığı şikayetler sırasıyla saç dökülmesi, kellik, saçlı deride kaşıntı ve yanma idi. Çalışmaya alınan 29 hastanın 24'ünde primer skatrisyel alopesi, beş hastada ise geçirilmiş inflamatuvar tinea kapitise sekonder skatrisyel alopesi vardı (Tablo 1).

Dermoskopik değerlendirmede saptanan vasküler yapılar, pigment ağ yapısı ve foliküler/perifoliküler özellikler Tablo 2'de gösterilmiştir.

Folikülitis dekalvansda (FD) foliküler açıklık kaybı dışında epidermal ve perifoliküler skuamlar, tufted follikülit ve kıvrımlı kırmızı düğümler azalan sıklıkta görüldü (Resim 1a,b). iki hastada foliküler püstül oluşumu vardı.

Brocq'un psödopeladında görülen en belirgin bulgu foliküler açıklık kaybı ve yapısız beyaz renkli alanlardı (Resim 2a). Bal peteği pigment paterni, dallanan kırmızı çizgiler ve epidermal/perifoliküler skuam diğer bulgulardı (Resim 2b).

Liken planopilarisli (LPP) tüm hastalarda foliküler açıklık kaybı ve perifoliküler beyaz renkli skuamlar belirgindi (Resim 3a,b). Balpeteği pigment paterni ve beyaz benekler dikkat çeken diğer bulgulardı.

Diskoid lupus eritematozuslu (DLE) her iki hastada foliküler açıklık kaybı ve dallanan kırmızı çizgiler vardı. Aktif DLE'si olan bir hastada foliküler kırmızı ve sarı benekler vardı (Resim 4a,b).

\section{Tablo 1. Çalışmaya katılan hastaların demografik özellikleri, klinik tanı ve hastalık süreleri}

\begin{tabular}{|l|l|l|l|l|}
\hline & $\mathbf{N = 2 9}$ & Kadın/Erkek & Ortalama yaş & Hastalık süresi (ortalama) \\
\hline Folikülitis dekalvans (FD) & 8 & $1: 7$ & 33 & 3 ay-7 yıl $(2,09 \mathrm{yll})$ \\
\hline Brocq'un psödopeladı & 7 & $5: 2$ & 33,4 & $1-20 \mathrm{yll}(7,8 \mathrm{yıl})$ \\
\hline Liken planopilaris (LPP) & 6 & $5: 1$ & 38,6 & $3 \mathrm{ay}-2 \mathrm{yıl}(0,9 \mathrm{yll})$ \\
\hline Tinea kapitis & 5 & $3: 2$ & 17,4 & $1-10 \mathrm{yll}(4 \mathrm{yll})$ \\
\hline Diskoid lupus eritematozus (DLE) & 2 & $1: 1$ & 34,5 & $6-20 \mathrm{yıl}(13 \mathrm{yıl})$ \\
\hline Dissekan selülit & 1 & $0: 1$ & 22 & $1 \mathrm{yıl}$ \\
\hline
\end{tabular}

Tablo 2. Skatrisyel alopesili hastalarda görülen dermoskopik paternlerin hastalıklara dağılımı

\begin{tabular}{|c|c|c|c|c|c|c|}
\hline & $\begin{array}{c}\text { Folikülitis dekalvans } \\
n=8(\%)\end{array}$ & $\begin{array}{c}\text { Brocq'un psödopeladı } \\
n=7(\%)\end{array}$ & $\begin{array}{c}\text { LPP } \\
n=6(\%)\end{array}$ & $\begin{array}{c}\text { DLE } \\
\mathrm{n}=2(\%)\end{array}$ & $\begin{array}{c}\text { Tinea kapitis } \\
n=5(\%)\end{array}$ & $\begin{array}{c}\text { Dissekan selülit } \\
n=1(\%)\end{array}$ \\
\hline Kıvrımlı kırmızı düğümler & $6(75)$ & 0 & 0 & 0 & 0 & 0 \\
\hline Dallanan kırmızı çizgiler & $5(62,5)$ & $3(42,8)$ & $2(33,3)$ & $2(100)$ & $2(40)$ & $1(100)$ \\
\hline Bal peteği pigment paterni & $3(37,5)$ & $4(57,14)$ & $4(66,6)$ & $1(50)$ & $1(20)$ & 0 \\
\hline Beyaz benekler & $4(50)$ & $2(28,57)$ & $4(66,6)$ & 0 & 0 & 0 \\
\hline Epidermal skuam & $7(87,5)$ & $3(42,8)$ & $2(33,3)$ & $1(50)$ & $3(60)$ & 0 \\
\hline Perifoliküler skuam & $7(87,5)$ & $3(42,8)$ & $6(100)$ & $1(50)$ & 0 & 0 \\
\hline Foliküler açıklık kaybı & $8(100)$ & $7(100)$ & $6(100)$ & $2(100)$ & $5(100)$ & $1(100)$ \\
\hline Tufted follikülit & $7(87,5)$ & 0 & 0 & 0 & 0 & 0 \\
\hline
\end{tabular}


Geçirilmiş inflamatuvar fungal infeksiyona sekonder skatrisyel alopesisi olan hastalarda foliküler açıklık kaybı, pembe-beyaz yapısız alanlar ve epidermal skuamlar saptanan en belirgin bulgulardı (Resim 5).

Dissekan selüliti olan bir hastada dallanan kırmızı çizgiler, sarı benekler ve foliküler açıklık kaybı görüldü.

\section{Tartışma}

Dermoskopi benin ve malin melanositik lezyonların tanısında, ayrıca deri kanseri ve diğer inflamatuvar, infeksiyöz dermatozların farklı tiplerinin saptanması ve ayırımında yararlı bir yöntemdir. Son yıllarda, dermoskopinin saç ve saçlı deri hastalıklarının tanı ve takibinde belirgin fayda sağladığı saptanmıştır ${ }^{8}$. Skatrisyel olmayan alopesilerin karakteristik dermoskopik özellikleri ile ilgili giderek artan sayıda
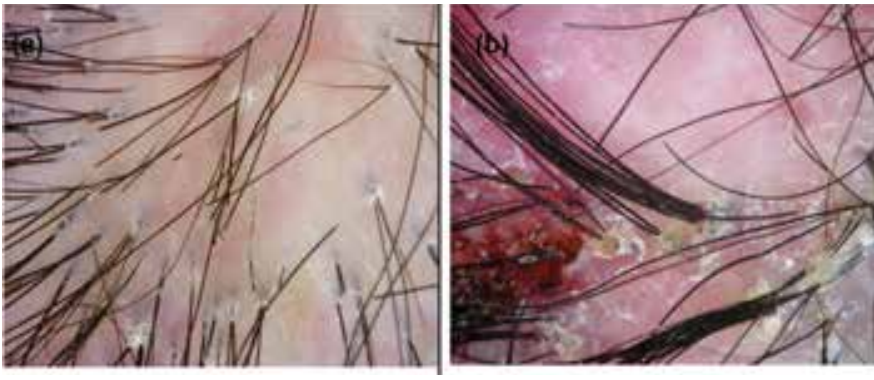

Resim 1. Folikülitis dekalvansda (a) perifoliküler skuam, foliküler açıklık kaybı ve yapısız beyaz alanlar, (b) tufted folikülit
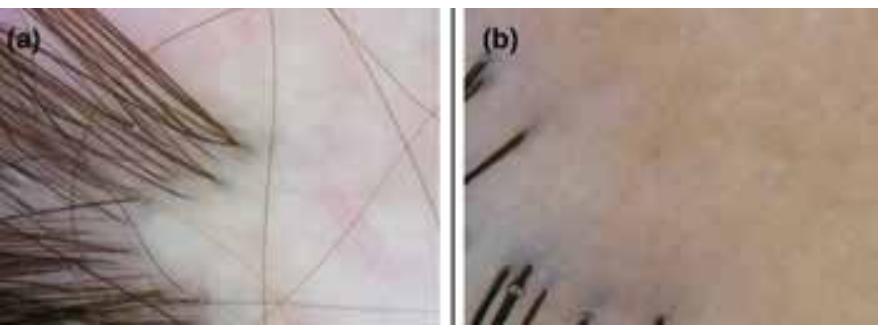

Resim 2. Brocq'un psödopeladında (a) yapısız fildişi-beyaz renkli alanlar, (b) bal peteği pigment paterni.
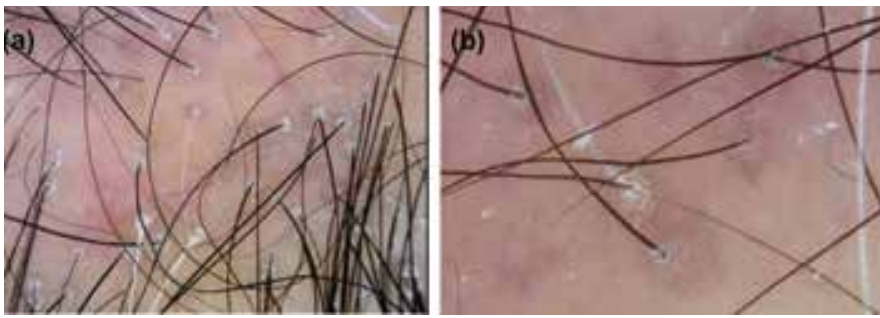

Resim 3. Liken planopilarisde (a) perifoliküler skuam ve balpeteği pigment paterni, (b) perifoliküler mavi-gri noktalar

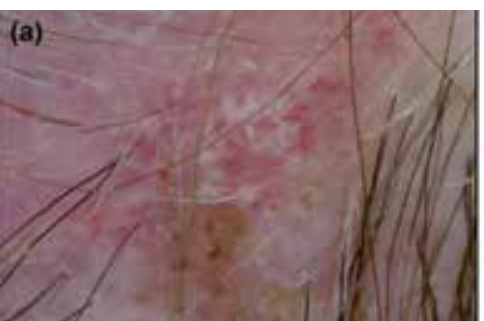

Resim 4. Diskoid lupus eritematosuzda tıkaçlar, (b) foliküler kırmızı noktalar kanıtlar olmasına rağmen, skatrisyel alopesinin ayırıcı tanısında saçı deri dermoskopisinin (trikoskopi) yararlılığı henüz kısmen incelenmiştir8-10. Biz, daha önceki yapılmış çalışmalara benzer bulguları elde ettiğimiz bu çalışmada klinik ve histopatolojik bulgulara göre skatrisyel alopesisi olan 29 hastayı değerlendirdik. Hastaların muayenesinde çılak gözle görülen özellikler dermoskopi ile daha basit ve kolay değerlendirildi. Skatrisyel alopesinin tipik bulgusu foliküler açıklık sayısında azalma tüm vakalarımızın dermoskopik değerlendirmesinde mevcuttu. Bu durum klinik olarak kalıcı alopesi ve histopatolojik olarak kıl folikülünün fibrozisi ile karakterizedir7,11

Saçlı derinin rekürren papülopüstüler lezyonları ile giden folikülitis dekalvansda, foliküler yıkım olduğunda inflamasyon geriler ve lezyon skatrisyel alopesiyle sonuçlanır ${ }^{12}$. Çalışmamızda, bu hastaların dermoskopik değerlendirmesinde foliküler açıklıklarda azalma ile birlikte epidermal ve peripilar skuam, çok sayıda kıvrımlı kapiller düğüm ve tufted follikülit sık görülen bulgulardı. Bazı yazarlar FD'da karakteristik vasküler bulguların olmadığını savunsa da, Ross ve ark. aktif hastalıkta epidermal ve infindubular akantozis ile ilişkili görülen interfoliküler kıvrımlı kapiller düğümlerin, alttaki süpürasyona reaktif bir fenomen olabileceğini vurgulamıştır9,13,14. Skatrisyel alopesiye sekonder olarak gelişen tek bir foliküler açıklıktan çıkan çok sayıda kılların varlığı (tufted folikülit) ise son yıllarda FD'ın ayırıcı özelliği olarak değerlendirilmektedir9,12,14.

Brocq'un psödopeladında dermoskopik özellikler tipik değildir ve tanı hem klinik hem de dermoskopik olarak diğer nedenlerin dışlanmasıyla koyulur ${ }^{14}$. Çalışmamızda foliküler açıklıkların görülmediği beyaz alanlar dışında bal peteği pigment paterni bu hastalıkta en sık görülen bulguydu. Bal peteği pigment paterni saç kaybı sonucunda kronik güneş maruziyetinin olduğu alopesik alanlarda görülür8,13. Çoğunlukla yavaş ilerleyen alopesik durumlarda görülen bu patern kronik hastalığın bir işareti olarak değerlendirilmektedir ${ }^{13}$

Skatrisyel alopesinin en sık nedeni olan LPP' de diğer çalışmalarla uyumlu olarak perifoliküler skuam, foliküler açıklık kaybı ve beyaz noktalar en sık görülen dermoskopik bulgulardı8,9,12. Perifoliküler tubuler karakterde gümüş beyazı skuamlar Rokowska ve ark. ' $\left.\right|^{9}$ tarafından LPP için tipik olarak değerlendirilmiştir. Ayrıca Duque-Estrada ve ark.'nın8 tanımladığı histopatolojik olarak interfaz dermatitinde pigment inkontinansıyla uyumlu mavi-gri noktaların, fibrotik folikülü çevreleyen hedef benzeri görünümü LPP'li bir olgumuzda görüldü.

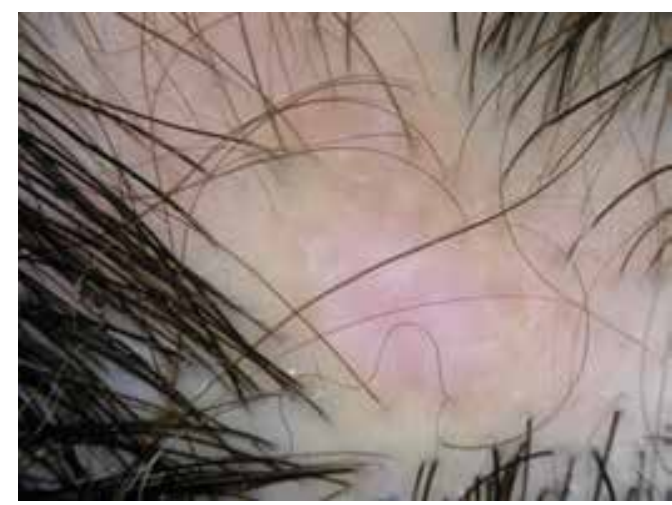

Resim 5. Sekonder skatrisyel alopeside foliküler açıklıkların görülmediği pembe-beyaz yapısız alanlar 
Foliküler açıklıklarda azalma dışında, bazal hücreli karsinom için tipik olan kalın dallanan damarlar DLE'li her iki olgumuzun dermoskopik değerlendirmesinde mevcuttu. Tosti ve ark.'15 tanımladığı foliküler kırmızı noktalar bir aktif DLE'li olgumuzda saptandı. Bu patern ekstravaze eritrosit ve dilate damarlarla çevrili dilate infundibulumla ilişkili bulunmuş ve aktif hastalığın tipik özelliği olarak belirtilmiştir9,15. Bunların dışında DLE için karakteristik olan foliküler keratotik tıkaçla uyumlu sarı benekler yine aktif DLE'si olan hastamızda görüldü. DuqueEstrada ve ark. ${ }^{8}$ koyu tenli DLE'li hastalarda pigment inkontinansıyla ilişkili benekli paternde mavi-gri noktaları tanımlamışlardır. Bu özellik deri fototipi II ve III olan hastalarımızda görülmedi.

Rakowska ve ark. sarı yapısız alanlarıve içinde distrofik kılların bulunduğu "3D" yapısındaki sarı noktaları dissekan selülitte saptamışlardır9. Biz de dissekan selülitli olgumuzda foliküler açıklıklarda azalma ile birlikte dallanan ince damarlar ve sarı benekleri dermoskopik değerlendirmede saptadık.

Anamnezinde geçirilmiş fungal infeksiyon öyküsü olan, alopesik alandan yapılan histopatolojik değerlendirmede ise pilosebase ünitede/retiküler dermisde fibrozis saptanan sekonder skatrisyel alopesili hastalarımızda foliküler açıklıkların kaybolduğu pembe-beyaz renkli alanlar vardı. Infeksiyonun aktif döneminde virgül benzeri kılların varlığı Slowinska ve ark. tarafından tinea kapitisin en belirgin ve ayırt edici özelliği olarak tanımlanmıştır. Ancak inflamasyon kıl foliküllerinde kalıcı hasara yol açarsa skar lezyonlarının geliştiği son evrede diğer nedenlere bağlı skatrisyel alopesilerden dermoskopik olarak ayırt edilmesi mümkün değildir16,17.

Sonuç olarak saçlı deri dermoskopisi hastalığın aktif döneminde skatrisyel alopesinin ayırıcı tanısına yardımcı, kolay ve hızlı uygulanabilen, invaziv olmayan bir yöntemdir. Ayrıca hekimi basit klinik inspeksiyonun ötesinde karakteristik dermoskopik bulgularla ileri değerlendirme konusunda uyarıp, harekete geçirebilir. Ancak bu yöntemin daha iyi anlaşılması için histopatolojik korelasyonun da yapıldığı geniş serili çalışmalara intiyaç vardır.

\section{Kaynaklar}

1. Otberg N, Wu WY, McElwee KJ, Shapiro J: Diagnosis and management of primary cicatricial alopecia: part I. Skinmed 2008;7:19-26.

2. Sullivan JR, Kossard S: Acquired scalp alopecia. Part I: A review. Australas J Dermatol 1998;39:207-21.

3. Headington JT: Cicatricial alopecia. Dermatol Clin 1996;14:773-82.

4. Ohyama M: Primary cicatricial alopecia: recent advances in understanding and management. J Dermatol 2012;39:18-26.

5. Inui S, Nakajima T, Nakagawa K, Itami S: Clinical significance of dermoscopy in alopecia areata: analysis of 300 cases. Int J Dermatolol 2008;47:688-93.

6. Wallace MP, Berker DA: Hair diagnoses and signs: the use of dermatoscopy. Clin Exp Dermatol 2008:35:41-6.

7. Inui S, Nakajima T, Itami S: Scalp dermoscopy of androgenetic alopecia in Asian people. J Dermatol 2009;36:82-5.

8. Duque-Estrada B, Tamler C, Sodré CT, Barcaui CB, Pereira FB: Dermoscopy patterns of cicatricial alopecia resulting from discoid lupus erythematosus and lichen planopilaris. An Bras Dermatol 2010;85:179-83.

9. Rakowska A, Slowinska M, Kowalska-Oledzka E, et al: Trichoscopy of cicatricial alopecia. J Drugs Dermatol 2012;11:753-8.

10. Kossard S, Zagarella S: Spotted cicatricial alopecia in dark skin. A dermoscopic clue to fibrous tracts. Australas I Dermatol 1993;34:49-51.

11. Inui S: Trichoscopy for common hair loss diseases: algorithmic method for diagnosis. J Dermatol 2011;38:71-5.

12. Tosti A: Dermoscopy of hair and skalp disorders with clinical and pathological correlations. London, Informa Heathcare, 2007;98-123.

13. Ross EK, Vincenzi C, Tosti A: Videodermoscopy in the evaluation of hair and skalp disorders. J Am Acad Dermatol 2006;55:799-806.

14. Rudnicka L, Olszewska M, Rakowska A, Slowinska M: Trichoscopy update 2011. J Dermatol Case Rep 2011;5:82-8.

15. Tosti A, Torres F, Misciali C, Vincenzi C: Follicular red dots. A novel dermoscopic pattern observed in scalp discoid lupus erythematosus. Arch Dermatol 2009;145:1406-9.

16. Slowinska M, Rudnicka L, Schwartz RA, et al: Comma hairs: A dermoscopic marker for tinea capitis. J Am Acad Dermatol 2008;59:77-9.

17. Mirmirani P, Willey A, Chamlin S, Frieden IJ, Price VH: Tinea capitis mimicking cicatricial alopecia: what host and dermatophyte factors lead to this unusual clinical presentation? J Am Acad Dermatol 2009;60:490-5. 